\title{
Electronic structure of fcc and bec close-packed silver surfaces
}

\author{
Moncef Said, František Máca, ${ }^{*}$ Kyozaburo Kambe, and Matthias Scheffler \\ Fritz-Haber-Institut der Max-Planck-Gesellschaft, Faradayweg 4-6, D-1000 Berlin 33, Germany \\ Niels Egede Christensen \\ Max-Planck-Institut für Festkörperforschung, Heisenbergstrasse 1, D-7000 Stuttgart 80, Germany
}

(Received 19 July 1988)

\begin{abstract}
We report results of total energy and electronic structure calculations of fcc and bcc silver bulk and surfaces. We find that under normal conditions (positive pressure) bcc silver is not stable. However, if silver is grown with a volume per atom $>10 \%$ larger than that of the well-known fcc silver, the total energy of the bcc structure becomes below that of fcc silver. For bcc (110) a surface state is predicted at the Fermi level.
\end{abstract}

Modern deposition techniques make it possible to grow crystals (or crystalline layers) with interatomic distances and structures different than those known so far. Nevertheless, the surface properties of differently structured materials have not been studied or compared systematically so far.

Using the density-functional theory (DFT) we performed total-energy and electronic-structure calculations for fcc and bcc silver in order to investigate the electronic properties of a new (so far not known) crystalline material (bcc silver). In fact, recent experimental studies of Aristov, Bolotin, and Grazhulis ${ }^{1}$ indicated that silver on InSb(110) may grow in the bcc structure up to many layers of thickness. The structure identification was drawn on the basis of Auger-electron spectroscopy and the lowenergy electron diffraction (LEED) pattern of a multilayer silver film. No LEED intensity analysis was performed, so that the interlayer distance (normal to the surface) remains uncertain. The LEED pattern indicated a somewhat perturbed two-dimensional bcc (110) layer.

Figure 1 displays the results of self-consistent scalarrelativistic linear muffin-tin-orbital ${ }^{2}$ (LMTO) calculations for silver fcc and bcc. We use the local-density approximation (LDA) for the exchange-correlation functional $^{3}$ and the atomic-sphere approximation for the effective potential. The basis set includes partial waves up to $l=3$.

At zero temperature and pressure the stable structure is fcc. The theoretical lattice constant is $4.02 \AA$ which compares well to the experimental value of $4.08 \AA$ (Ref. 4) and to a previous self-consistent Korringa-Kohn-Rostoker calculation $^{5}$ which gave a lattice constant of $4.12 \AA$. The bec structure has only slightly higher energy. Its minimum energy is at a lattice constant of $3.2 \AA$. bcc silver with such lattice constant will not be stable but it will undergo a phase transition to the fcc structure. However, for negative pressure (which at least is a possible concept in theoretical studies) we find that the bcc totalenergy curve gets below the fcc curve. From the Gibbs construction (the common tangent on both fcc and bcc total-energy curves) we expect that the phase transition from fcc to bcc starts when the volume per atom is about $10 \%$ larger than that of the equilibrium volume of the fcc structure. At about $15 \%$ the bcc structure would be dominant. The corresponding lattice constant is $3.37 \AA$, which agrees with the value deduced by Aristov, Bolotin, and Grazhulis ${ }^{1}$ from their LEED pattern.

It seems that the above-mentioned "negative pressure" can be realized experimentally if silver is deposited on certain substrates that let it start growing with a bcc (110) layer, which is the closest-packed bcc layer. The next layer of silver atoms will then occupy fourfold (maximum coordination) sites. The self-consistent bulk calculations show that the interatomic distances of the fcc and the bcc structures (the latter at a atomic volume $15 \%$ larger than that of fcc) are practically the same, namely, $2.9 \pm 0.05$ $\AA$. Therefore it may be expected that this distance determines (in a first approximation) also the interlayer distance. As a consequence, this would give the threedimensional bcc structure. However, a slightly smaller interlayer spacing cannot be excluded. Therefore a LEED intensity analysis that allows determination of the interlayer spacing perpendicular to the surface would be desirable. We note that the fcc and bcc total-energy curves

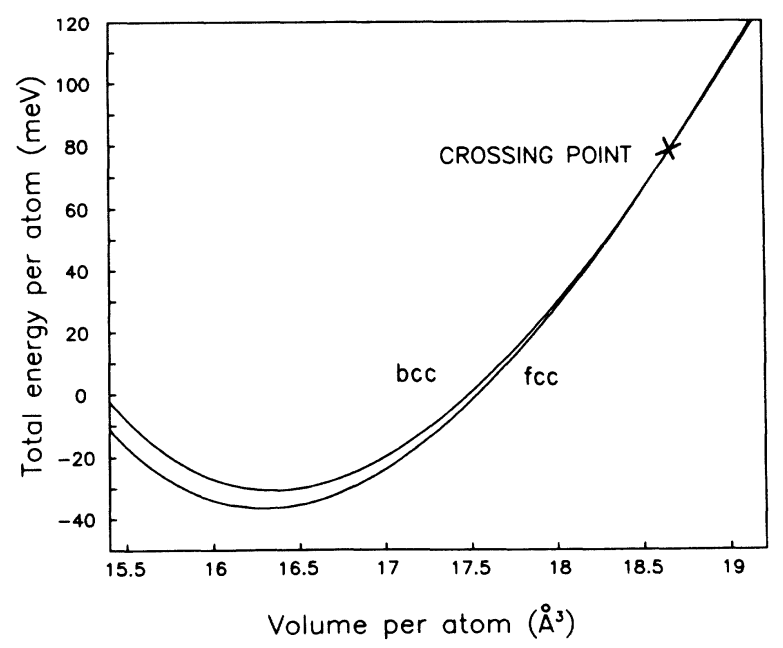

FIG. 1. Total energy per atom as a function of volume per atom for fcc and bec silver. 
have very similar slope at the crossing point. Therefore it may be difficult to grow pure bcc silver, i.e., to avoid surface defects, dislocations, and fec clusters. Further details, of our total-energy calculations including a discussion of the hcp structure, will be presented elsewhere. ${ }^{6}$

In particular we analyzed the differences between the fcc and bcc silver surfaces. Figure 2 displays the results for the $\mathbf{k}_{\|}$-resolved density of states for fcc (111) and bcc (110) surfaces which are the closest-packed surfaces of these two crystalline structures. Results for other faces will be discussed elsewhere. These calculations are performed using the layer-Green's-function method, ${ }^{7}$ which allows comparison of the surface and bulk properties in a consistent picture. It does not place a restriction on the spatial extent of surface-state (or surface-resonance) wave functions and gives detailed information about the electronic properties, such as $\mathbf{k}_{\|}$-resolved, layer-resolved, and angular-momentum-resolved densities.

For the present study we use the self-consistent (density-functional-theory, local-density approximation) potential for bulk fec silver of Morruzi, Janak, and Williams ${ }^{5}$ and took it for both our fcc and bcc studies. Calculations with the potentials of our linear muffin-tin-orbital method calculations give essentially the same results. The surface was approximated by a truncated bulk, using a simple step surface barrier. This step function touches the surface-layer muffin-tin spheres. Its height is $11 \mathrm{eV}$. This is a reasonable first approximation and already gives a good description of the surface density of states. Also, surface states are described (see below). Whereas their wave-function character is reliable, their exact energy position is somewhat uncertain. The latter depends on the details of the potential in the surface region. For the lattice constants we use $4.12 \AA$ for the fcc (see Ref. 5) and $3.37 \AA$ for the bcc structure. The intralayer scattering is described by spherical waves with $l \leq 3$. For the interlayer scattering we use 15 (fcc) and 21 (bcc) plane waves.

Figure 2 shows results for the $\mathbf{k}_{\|}$-resolved density of states for different layers at $\mathbf{k}_{\|}=(0,0)$. Of course, the peaks in the bulk density of states (row 4) correlate with the bulk band structure along the $\Gamma L$ (for fcc) and $\Gamma N$ (for bcc) directions. Note that the width of the $d$ bands is very similar in both structures; the bec $d$ band is only slightly narrower than the fcc $d$ band. If we go to the surface, we observe some changes in this density of states. The most important result is a surface state which we find for both systems (fcc and bcc silver) close to the Fermi level. The intensity of this surface state decreases rapidly from the first to the second to the third layer.

For fcc silver (111) this surface state is well known experimentally $^{8}$ and theoretically. ${ }^{9-12}$ It is strong at $\mathbf{k}_{\|}=(0,0)$ and shows a dispersion with increasing $\mathbf{k}_{\|}$to higher energies. As a consequence, this surface state can be seen in angle-resolved photoemission ${ }^{11}$ (occupied) as well as in inverse photoemission ${ }^{10,12}$ (empty). The agreement between our calculations and these experiments is nearly perfect.

As the existence of bcc silver is not yet established, nothing is known experimentally about the electronic structure of its (110) surface. The calculated energy position of the predicted surface state is right at the Fermi level. The accuracy of our calculation is estimated to be $\pm 0.3 \mathrm{eV}$. Therefore we cannot tell safely if this surface state is partly occupied with electrons or if it is empty.

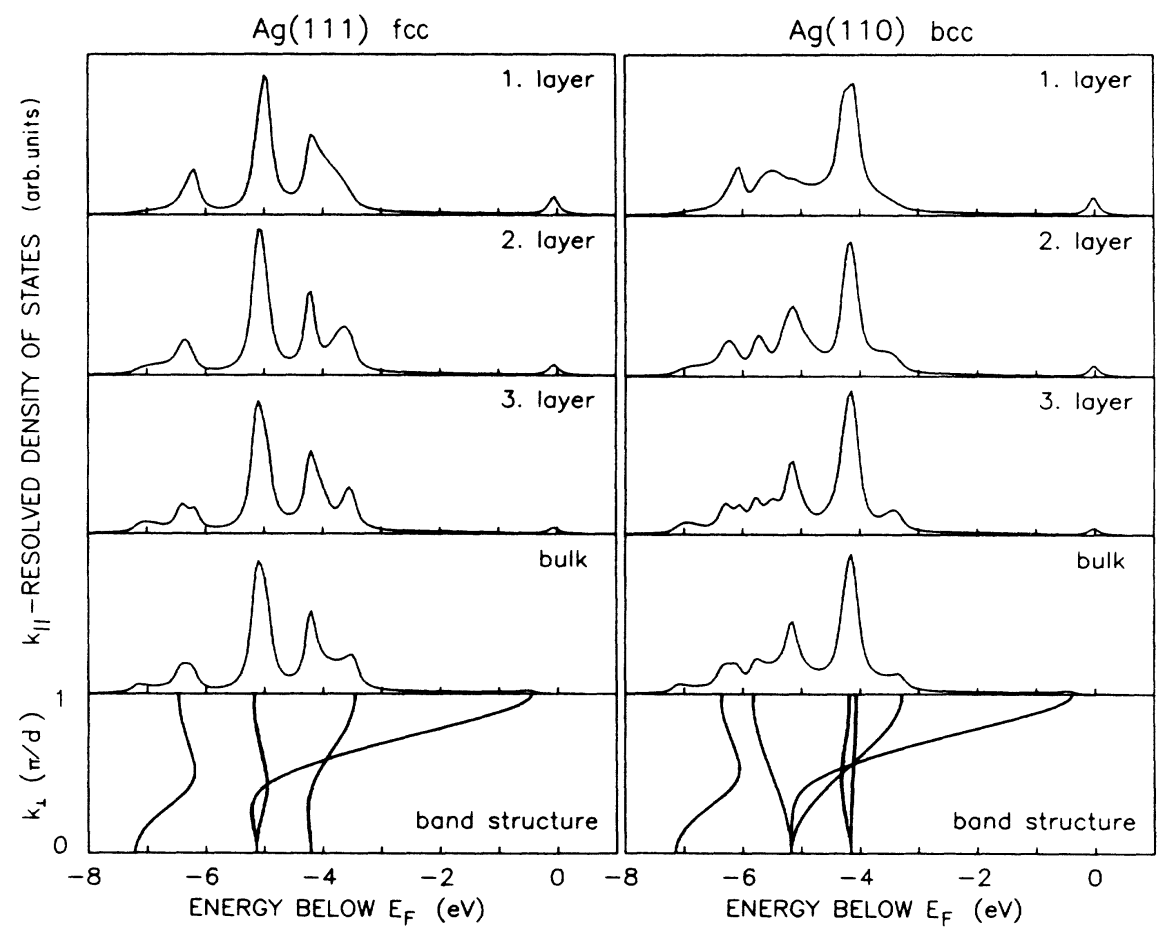

FIG. 2. $\mathbf{k}_{\|-}$and layer-resolved density of states for silver fcc $(111)$ and bcc $(110)$ for $\mathbf{k}_{\|}=(0,0)$. The bottom row gives the corresponding bulk band structure along the surface normal, which is $\Gamma L$ for fcc (111) and $\Gamma N$ for bcc (110). $d$ is the interlayer spacing. 
The nature of this surface state is very similar to that at the fcc (111) surface. In both cases the surface state is split off the bulk $s-p$ band at the Brillouin zone edge due to the repulsive surface barrier. In both cases the orbital character is $p_{z}$, where $z$ is the surface normal.

If the results of the $\mathbf{k}_{\|}$-resolved density of states are integrated over the surface Brillouin zone, we obtain the layer density of states (see Ref. 6). This shows a multistructured peak for the bulk density of states for the fcc silver $d$ band and a two-peak structure for bcc silver. These bulk densities of states are largely determined by the $d$ states at $L$ and $X$ (fcc) and $N$ (bcc). For the $d$-band energies at $L$ and $N$ see the lowest row of Fig. 2. This qualitative difference between the bulk densities of states of fcc (a broad peak) and bcc (two well-separated peaks) is a result of the tight-binding nature of $d$ bands and clearly distinguishes the two crystalline structures. Therefore both systems should be clearly distinguishable in absorption as well as photoemission experiments.
In conclusion, we performed self-consistent total-energy calculations for fcc and bcc silver that show that bcc silver is a possible crystalline material. It is, however, necessary to increase the volume per atom by $>10 \%$ compared to the atomic volume of standard conditions fcc silver. These results support the interpretation of Aristov, Bolotin, and Grazhulis ${ }^{1}$ who studied silver layers on InSb(110).

In particular, we discussed results of layer-Green'sfunction calculations of fcc (111) and bcc (110) silver surfaces. These results show that fcc and bcc silver should be clearly distinguishable by absorption measurements as well as by angle-resolved photoemission. For both surfaces, fcc (111) and bcc (110), we find a surface state right at the Fermi level. For fcc silver this is in good agreement with known experiments, whereas for bcc silver it is a prediction.

M. Said acknowledges financial support from the Alexander von Humboldt Stiftung.
*Permanent address: Institute of Physics, Czechoslovak Academy of Sciences, Na Slovance 2, 18040 Prague 8, Czechoslovakia.

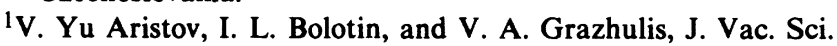
Technol. B 5, 992 (1987).

${ }^{2}$ O. K. Andersen, Phys. Rev. B 12, 3060 (1975).

${ }^{3}$ D. M. Ceperley and B. J. Alder, Phys. Rev. Lett. 45, 566 (1980).

${ }^{4}$ M. E. Straumanis and S. M. Riad, Trans. AIME 233, 964 (1965).

${ }^{5}$ V. L. Moruzzi, J. F. Janak, and A. R. Williams, Calculated Electronic Properties of Metals (Pergamon, New York, 1978).

${ }^{6}$ F. Máca, M. Said, N. E. Christensen, K. Kambe, and M.
Scheffler (unvublished).

${ }^{7}$ F. Máca and M. Scheffler, Comput. Phys. Commun. 38, 403 (1985); 47, 349 (1987); and (to be published).

${ }^{8}$ H. F. Roloff and H. Neddermeyer, Solid State Commun. 21, 561 (1977).

${ }^{9}$ K. M. Ho, C. L. Fu, S. H. Liu, D. M. Kolb, and G. Piazzia, J. Electroanal. Chem. 150, 235 (1983).

${ }^{10}$ S. L. Hulbert, P. D. Johnson, N. G. Stoffel, and N. V. Smith, Phys. Rev. B 32, 3451 (1985).

${ }^{11}$ J. G. Nelson, S. Kim, W. J. Gignac, and R. S. Williams, Phys. Rev. B 32, 3465 (1985).

${ }^{12}$ A. Goldmann, V. Dose, and G. Borstel, Phys. Rev. B 32, 1971 (1985). 